\title{
DEALING WITH KEY TERMS IN RISK ANALYSIS AND PHENOMENON OF UNCERTAINTY IN THIS PROCESS
}

Unifying key terms in crisis management is one of the main vague field when performing risk analysis. Differences between terms and their definitions should eventuate into problems with communication in this area. I will mention a causal relationship between danger, treat and risk and I will try to integrate them into the surrounding of technological processes. In the next paragraph I will be dealing with risk analysis which should be characterized as a systematic use of available information to determine how often specified events may occur and the magnitude of their likely consequences. Therefore the wide range of uncertainty will occur when performing risk analysis. The main sources of uncertainty were described in projects made by Joint Research Centre as a part of whole assurance project, known as benchmark studies made for two chemical and one nuclear power plants.

Key words: technological process, danger, treat, risk, risk analysis, uncertainty, benchmark studies

\section{Introduction}

Any technological or technical system can not be considered as a perfect one. Oftentimes we should consider machine, technological process or another technical equipment as a reliable but there is no way to consider human being as a certain reliable. Companies which dispose of risk technologies should have sufficient knowledge in systematic and complex risk assessment. But it is not possible to do it right in the case of insufficient information or differences in key terms: hazard, danger, risk. In part 2.1 I present my point of view on causal relationship between these terms. But when all things are well done there is still one thing resonating. When performing a risk analysis a wide range of uncertainties will be inevitably introduced during the process especially in qualitative phase mentioned in part 2.2. In part 2.3 there are mentioned benchmark studies which were performed by Joint Research Centre at Ispra and Risk National Laboratory which pointed to an increased awareness of the potential uncertainties in Risk analysis and highlighted a number of important sources of such uncertainties. These are the main fields I would like to deal with in my PhD studies.

\section{Uncertainty and Risk analysis}

Risk analysis is often considered as an identification of appreciated system and detection of danger, treat and risk in considered system [8]. When talking about risk analysis we mostly mean Quantitative risk analysis (QRA). In many engineering situations, most variables used in analysis will be associated with uncertainty. And the next important thing after ending risk analysis is to identify how the uncertainty affects results. The impacts and sources of such uncertainty were presented in benchmark studies realized on chemical establishments and nuclear power plant.

\subsection{Key terms resolution - danger, treat and risk}

This first paragraph presents causal relationship between terms danger, treat and risk and highlights that using these terms request a correct understanding of their definitions. If we unify definitions of these terms we will avoid problems of misunderstanding in many fields of risk management especially in risk communication. I would like to introduce these terms from my point of view to show causality among them when trying to integrate them into technological processes environment. Each technological process needs inputs to be operated and to reach aims of the company - products and services. Fig.1 shows a technological process which disposes dangerous substances or machines and therefore it is running through the system danger - treat - risk. A product or service should be considered as an output if everything goes right. In case a floater impacts on the system because of interior or exterior causes the emergency situation should occur. It can result into stopping the technological process if there is no possibility to return the system to the starting point or, on the other hand, to revalue arrangements which were done for hazard reduction. Somebody should have restrictions against terminology of the key terms but it has to be said that in this area many differences of definitions of the key terms in crises management have been occurring (Fig. 1).

It is important to mention causal relationship between the three terms. Danger is taken as a source of treat, it is latent character of an object, machine, activity or technological process. And

\footnotetext{
* Katarina Holla

Department of Crisis management, Faculty of Special Engineering, University of Zilina, Slovakia Republic, E- mail : katarina.holla@fsi.uniza.sk
} 


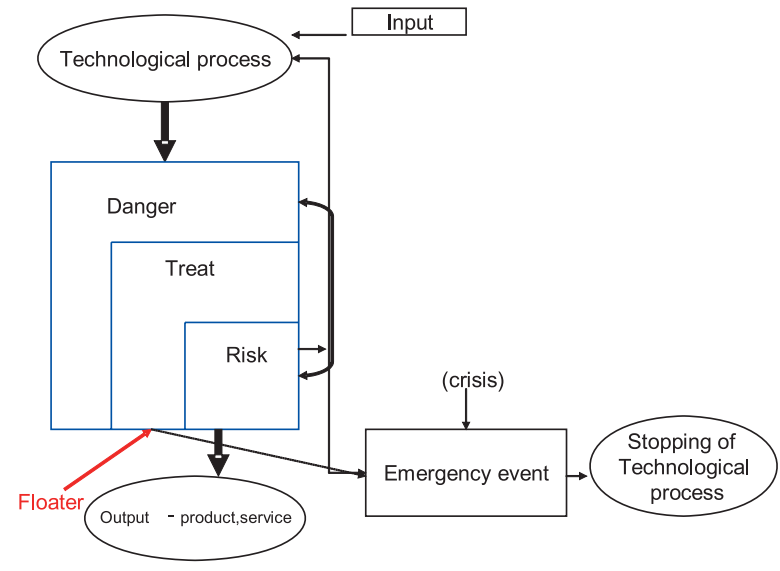

Fig. 1 Integration of danger, treat and risk into technological processes surrounding

treat is then a status where danger should be activated. Treat consequences has close relationship on probability that an undesirable event will happen and this relationship (correlation) is represented by "risk". Correct understanding of these words should eliminate uncertainty which results from misunderstanding and different point of view on their definitions.

\subsection{Uncertainty and Risk analysis}

Risk analysis is in general considered as a identification of appreciated system and detection of danger, treat and risk in considered system [8]. That is why I explained their role in the paragraph 2.1. However there are many definitions of risk analysis in area of risk management. One defines it as a systematic use of available information to determine how often specified events may occur and magnitude of their consequences [5]. Phases of risk analysis are: system description, danger identification, sce- narios and risk evaluation[6]. Results of any risk analysis are inevitably uncertain to some degree. Because of inevitable limitations of the risk analysis approach it must be acknowledged that the true risks could be higher or lower than estimated. In general, the word 'uncertainty' means that a number of different values can exist for a quantity [1]. The uncertainty should be divided into two categories: aleatory and epistemic. Aleatory stochastic uncertainty or due to randomness should result from bad knowledge of risk figures and their distribution, quantities such a failure rates, meteorological conditions at the time of release. Epistemic (reducible) is related to incomplete knowledge about phenomena of concern and inadequate matching available databases to the case under the assessment [1]. In addition, the application of different methods and methodologies will contribute to the total uncertainty of variability of risk analysis final outcome.

Fig. 2 shows gradualness of risk analysis where in each phase there are partial uncertainty and partial error increasing to the final $\mathrm{N}$ and final $\mathrm{E}$. Each phase is characterized by its own uncertainty and errors and input uncertainty and errors from the previous phase. Finally we need to count not just with results of risk analysis but also estimate the uncertainty related to the final figure.

\subsection{Benchmark studies}

As it is shown on Fig. 2 uncertainty could increase into considerable amount what can affect final result of risk analysis. These impacts and sources of uncertainty were presented in benchmark studies (benchmark exercises) performed by Joint Research centre (JRC) directly or with cooperation for two chemical and one nuclear power plants. These benchmark studies could serve as a guide for dealing with uncertainty for specialist in area of risk analysis processes. Some important outcomes of benchmark exercises of chemical establishments for West Europe and the other one for Eastern Europe are presented in text undermentioned.

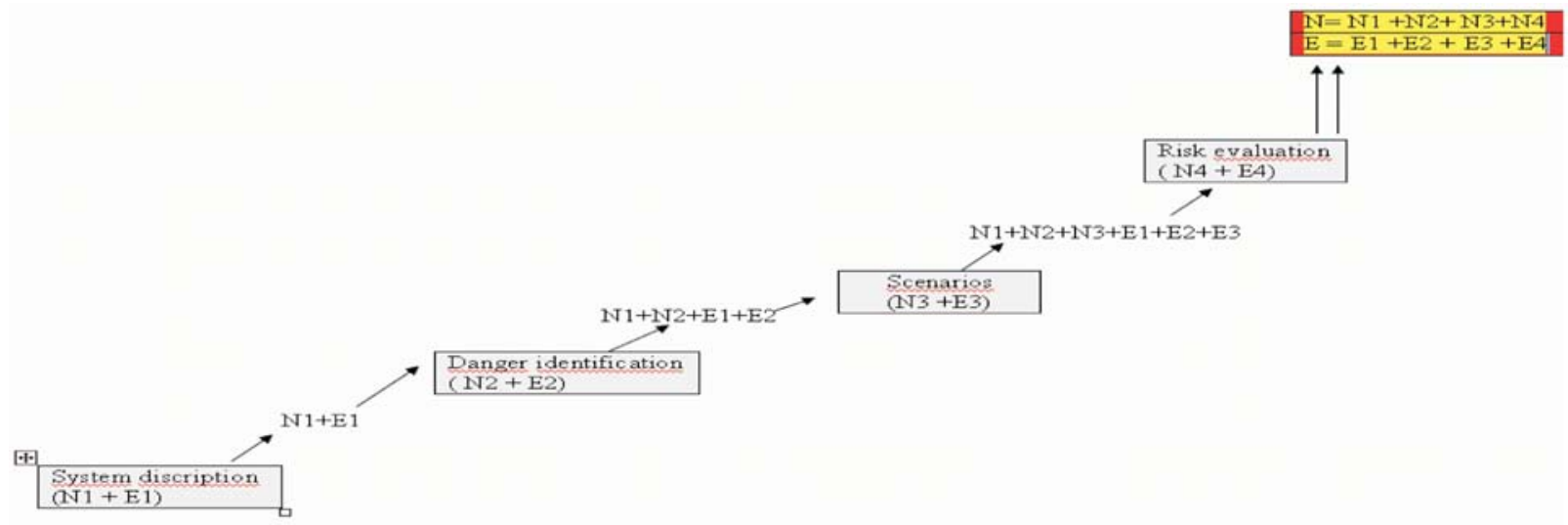

$N$ - uncertainties, E- error

Fig. 2 Cumulation of uncertainties in phases of risk analysis. 


\subsubsection{Assessment of Uncertainties in Risk Analysis of Chemical Establishment in West European countries (Benchmark exercise)}

Aniello Amendola and his team presented the assurance project called "Assessment of Uncertainties in Risk Analysis of Chemical Etablishments". Seven teams performed risk analysis for the same chemical facility, an ammonia storage. This project was made by the Joint Research Centre at Ispra and Risk National Laboratory. The research was accomplished through coordinated exercise and led to the comparison of results in order to reveal the causes for differences between the partners results. The results point out awareness of the potential uncertainties in risk analysis and highlight a number of important sources of such uncertainties. The research was divided into a documentation phase (description of a plant), qualitative analysis phase (hazard identification and qualitative risk ranking), quantitative analysis phase (quantitative risk analysis), and implication of uncertainties in risk assessment to risk informed decision. The hazard identification phase uncovered that the ranking of hazardous scenarios by probabilistic and deterministic approach imposed completely different conclusions. In spite of a big difference in frequency assessment of the same hazardous scenarios, there was good consensus on the ranking among the adhere of the probabilistic approach. There were found large differences in the frequency assessment as well as in the assessment of consequences. This assessment could serve as a guide to areas where caution must be taken when performing risk analysis [1].

\subsubsection{Benchmark Exercise in Quantitative Area Risk Assessment in Central and Eastern European Countries (Benchmark exercise)}

In order to better understand the risk analysis practices and methodologies adopted in the Accession and Candidate Countries, it was agreed to launch a third benchmark exercise focusing on the evaluation of the risk of a particular area in the proximity of a hazardous establishment. This was the next project made by the Joint Research Centre for Central and Eastern European Countries. The project was structured in three main phases: a documentation phase and two working phases. The countries involved in this research were: Bulgaria, Cyprus, Hungary, Latvia, Lithuania, Poland, Romania, Slovenia, and Slovakia. The report was launched in 2007. The main intention was to offer a general perspective on how independent reviews of the same risk analysis study conducted from the competent authority's standpoint might differ from each other and be reflected in a different evaluation of the risk of a certain Seveso type establishment. The outcome of such an analysis has the evident advantage of contributing towards better understanding the inspection criteria and current practices used by the different national authorities [4].

\section{Conclusion}

For all the reasons already mentioned I would like to point out the requirement of unifying the key words (danger, treat and risk) which is one of the main steps to avoid mistakes in the area of risk communication and to create appropriate field for risk analysis. As a part of it, risk analysis is inevitably uncertain to some degree what can bring in some problems in final results. And there is a question how issues of uncertainty are dealt with in existing safety regulations and in existing standards for risk analysis and management. I would like to highlight the fact that there is a big need to deal with uncertainty and to count with it in risk analysis and benchmark studies could serve as a guide to areas where caution must be taken when performing risk analysis. Slovak legislation does not consider impacts of such uncertainties in area of risk management and risk analysis. From my point of view it is necessary to incorporate this phenomenon into area of risk management.

\section{References}

[1] AMENDOLA, A., LAURIDSEN, K., KOZINE, I., MARKERT, F. , CHRISTOU, M., FIORI, M.: Assessment of Uncertainties in Risk Analysis of Chemical Establishment, The Assurance report, Final summary report, Denmark 2002, p. 49, ISBN 87- 550 - 3063 -7.

[2] PACAIOVA, H.: Appraisal of risks - Comparison of Method Definitions and Advances (in Slovak), Motivacia k panelovej diskusii, Forum BOZP 2003, Krpacovo, 2003.

[3] SIMAK, L.: Crisis Management in Public Administration (in Slovak), ZU Zilina 2004, ISBN 80-88829-13-5.

[4] FABBRI, L., JIRSA, P., CONTINI, S.: Benchmark Exercise in Quantitative Area Risk Assessment in Central and Eastern European Countries (BEQUAR), Final report. Institute for the Protection and Security of the Citizen, Italy, 2007.

[5] www.pce.govt.nz/reports/pce_reports_glossary.shtml, 9. 3. 2007.

[6] DANIHELKA, P.: Modul 3 level 3 - Why Risk management, Power point presentation, Ostrava, 2005.

[7] SINAY J.: Risks of technical maintenances - Managing of risks (in Slovak), OTA, a.s. Kosice. 1997. ISBN 80-967783-0-7.

[8] http://dict.die.net, 9. 3. 2007.

[9] http://www.mindtools.com/pages/article/newTMC_07.htm, 15. 10. 2007. 